\title{
A dynamic splint for the treatment of spasticity of the hand after stroke? Recognition of its design, functionality and limitations: A narrative review article.
}

\author{
Bianca $\mathrm{C}^{1,2}$, Machuki JO', Chen $\mathrm{W}^{2 *}$, Zhang $\mathrm{M}^{2}$, Shang $\mathrm{K}^{2}$ \\ ${ }^{1}$ Xuzhou Medical University, Tongshan road, Xuzhou, P.R. China \\ ${ }^{2}$ Xuzhou Rehabilitation Hospital, P.R. China
}

\begin{abstract}
Occurrence of spasticity of the hand after a stroke event is recognized as a major cause of functional limitations which reduces the quality of life in patients. Dynamic splints are among the alternative rehabilitation treatments for reducing spasticity of the hand. However, evidence supporting their effectiveness in restoring hand functionality, their long-term and short-term effects is limited. Moreover, standardized methods of designing dynamic splints are currently lacking. This review describes the methods used to design dynamic splints, their functionality and the current issues surrounding their application to post-stroke patients with spasticity of upper limbs. In so doing, we provide the foundational knowledge which may be tested in future clinical trials and in splint edifications to enhance the efficiency of splints to improve the quality of life in stroke patients. When properly designed to fit a patient, a dynamic splint can promote the functional recovery of a spastic hand. Further research is required to deepen our understanding on the clinical utility of dynamic splints in post-stroke patients to optimize their recovery outcomes.
\end{abstract}

Keywords: Spasticity, Dynamic splint, Upper limb, Stroke, Hand.

Abbreviations: CMC-Carpometacarpal; CVA-Cerebrovascular disease; C5-Cervical spine 5; DIP-Distal Interphalangeal; EMGElectromyography; g-gram; IP-Interphalangeal; MAS-Modified Ashworth Scale; MCP-Metacarpophalangeal; PPE-Personal Protective Equipment; PIP-Proximal Interphalangeal; T2-Thoracic spine 2

Accepted on December 20, 2018

\section{Introduction}

Stroke/Cerebrovascular disease (CVA) is a condition that is characterised by interruption of any part of the cerebral circulatory system caused by occlusion or rupture of blood vessels [1]. CVA is the $4^{\text {th }}$ most common cause of death and the main cause of disabilities occurring in mid to late adulthood world-wide [1-3]. After a lesion occurs in the structures of cerebral circulation, spasticity of upper limbs or lower limbs develops depending on the area of cerebral circulation affected $[4,5]$. In post-stroke patients, spasticity frequently occurs in the upper limbs because the middle cerebral artery is the largest artery of the brain and it is the most affected artery by strokeevoked lesions [6,7]. Spasticity is defined as a motor disorder characterized by a velocity-dependent increase in tonic stretch reflexes/muscle tone with exaggerated tendon jerks, resulting from hyperexcitability of the stretch reflex, as one component of the upper motor neuron syndrome [8]. Other authors suggest that it is a sensorimotor phenomenon related to the incorporation of the nervous system motor responses to sensory input and it does not occur alone [9]. Instead it coexists with a myriad of other characteristics such as contractures, hypertonia, muscle weakness and movement disorders [9].

Upper limb spasticity poses functional challenges to a poststroke patient when engaging in daily living activities [7]. If not treated it causes learnt non-use, muscle weakness, muscle atrophy, poor hand functional skills and sensory loss in the affected upper limb [7]. Rehabilitation professionals design and adapt dynamic splints which are meant to maintain muscle tissue length in stroke patients with spasticity [10]. A dynamic splint is defined by Dyna Splint Systems Inc [1] as a twosided non-circumferential spring loaded tensioning apparatus that increases joint range of motion by providing a low-load prolonged timed tissue stretch. Splints have been used for stroke patients since 1911 [11,12]. This review focuses on the design of a dynamic splint, its functionality, the current issues and limitations surrounding its usage in post-stroke patients with spasticity of upper limbs.

\section{Literature Review}

\section{The typical patterns of hand spasticity resulting from stroke}

Stroke affects the motor cortex in the internal capsule thereby producing initial hypotonia and absent tendon jerks followed weeks later by spasticity in antigravity muscles of the upper limb [13]. The initial paresis after stroke is caused by formation of lesions, neuronal destruction, cerebral oedema, increased cerebral inhibitory activity and ischemia [13]. The mechanism by which spasticity emerges in the upper limb is not fully understood but some processes have been suggested to be involved including hyperactive alpha motor neurons in muscles, 
Citation: Bianca C, Machuki JO, Chen W, et al. A dynamic splint for the treatment of spasticity of the hand after stroke-recognition of its design, functionality and limitations: A narrative review article. J Neurol Neurorehabil Res. 2018;3(2):1-7.

abnormal excitability of spinal interneurons, loss of supraspinal inhibitory or excitatory influences and intra-muscular changes in the affected limb $[9,14,15]$. After formation of lesions, the brain compensates for normal movements using synergies [16]. The simultaneous presence of synergies, coactivation of agonist and antagonistic muscles result in abnormal postures which makes it difficult for the patient to perform voluntary movements of the hand. Such patients experience flaccidity, spasticity or weakness in the shoulder muscles, especially abductors, wrist muscles and finger extensors [16].

Spasticity of the upper limbs is a major outcome after stroke [1719]. The hand spasticity pattern also known as 'flexor synergy' is characterized with a posture that consists of internal rotation and adduction of shoulder combined with flexion at the elbow, wrist and finger joints [12,20]. This causes unequal forces between the agonist and antagonist muscles of the upper limbs thereby compromising the static joint position and dynamic limb movements [20]. Pain and motor pathways influence each other which produces spasticity and pain concurrently [15]. The functionality of the hand is diminished because of the inability to isolate the appropriate agonist muscle and increased muscular force at task-unrelated muscles [16]. On this basis recovery of a spastic hand depends on the reduction of cerebral oedema, necrotic tissue and reorganisation of the central nervous system [21].

\section{Anatomical considerations of the hand in relation to dynamic splinting}

Understanding the anatomical structures of the forearm, wrist and hand is important to the designing a splint [22]. Some of the important anatomical structures and pressure points to consider include the palmar creases of the hand (distal, proximal, thenar and wrist), arches of the hand (distal; longitudinal and proximal transverse), styloid processes (radial and ulnar),finger web spaces and joint intersections (MCP; CMC; PIP; DIP and IP of the thumb) [22]. The knowledge of normal joint functions and kinematic movements such as rolling, gliding and spinning is also essential during the design of a dynamic splint with high stability and mobility [23]. A human hand is made up of the wrist; palm and fingers [24]. It is the most flexible part of the human skeleton and it exhibits fine motor skills when performing daily living activities [24].

The hand consists of 27 bones, 27 joints, 34 muscles, over 100 ligaments and tendons as well as numerous blood vessels and nerves [24]. The back of the hand is called the dorsal side and the palm of the hand is called the volar side [25]. The 27 bones are divided into 8 carpal bones (wrist area); 5 metacarpal bones (palmar area) and 14 phalanges (finger area) [25]. There are 5 metacarpal bones which serve as the foundations for the thumb; index; middle; ring and little fingers. The 14 phalanges serve as the fingers with proximal (near to palm); medial (middle) and distal parts (near to nails) [25]. The thumb consists of 2 phalanges while each of the other 4 fingers consists of 3 phalanges [24]. The joints of a hand include the carpometacarpal CMC (wrist area), metacarpal MCP (knuckle area), proximal interphalangeal PIP (in finger near palm area), distal interphalangeal DIP (in finger near nails) and only one interphalangeal IP joint in thumb [25].

In addition; the hand contains intrinsic (thenar, deep palm and hypothenar area) and extrinsic (dorsal and volar side) muscle groups [25]. Innervation of the hand is supplied by the spinal root nerves from C5-T2 in the form of radial (lateral 3 and half fingers on dorsal side), median (lateral 3 and half fingers volar side) and ulnar nerves (medial one and half fingers) [24]. Blood supply to the hand originates from the radial artery (which runs in front of wrist and thumb area) where a pulse can be taken and the ulnar artery (which runs along the ulnar nerve distribution area) [26]. The functional position of the hand is achieved when the wrist is in 20-35 degrees of extension; normal transverse arch; PIP joints flexed from 45-60 degrees and the thumb in abduction combined with opposition [22]. This position allows the hand to perform tasks using power grips such as cylindrical; spherical and hook or precision grips such as pad-to-pad; tipto-tip; pad-to-side; side-to-side and lumbrical [24]. The normal osteo-kinematics of the hand include movements of fingers such as extension; flexion; adduction and abduction; while thumb movements include extension, flexion, adduction, abduction and opposition [27].

\section{An overview of dynamic splints}

Basic types of splints for the hand: A splint is a noncircumferential external device made from different materials and it is designed to apply; distribute or remove forces to/from the body in a controlled manner to either maintain movement; change shape of muscle tissue or reduce spasticity [28]. The static (immobile) and dynamic (mobile) splints are the common types of splints used for the hand [28]. Common hand static splints include ulnar gutter, radial gutter, thumb Spica, volar, dorsal, single and double sugar tong [28]. This review will be focused on dynamic splints. In comparison to a static splint; a dynamic splint is made from the same materials as a static splint but contains extra components which allow mobility e.g., outrigger; springs; elastic strings and individual finger belt. An outrigger is a projection from the splint base which is used by the therapist to position the mobilizing force of the splint to the patient's limb to allow tissue remodelling and improve hand function [12]. Both types of splints perform similar functions although a dynamic splint is superior as it provides more benefits such as reduction of spasticity; allowing comfortable stretch; increasing compliance and motivation; reduction of joint pain, prevention of oedema, repositioning fingers into extension positions and increasing brain plasticity [29].

Dynamic splinting materials for the hand: The choice of splinting materials depends on their memory; drapability; elasticity; ability to bond; time to heat; flexibility; durability and thickness [6]. Dynamic/static splints can be made from different materials such as high/low temperature thermoplastics (Polypropylene, Polymethyl methacrylate, low/high density Polythene, Polyform ${ }^{\mathrm{TM}}$; Prodrape-TTM, Ezeform ${ }^{\mathrm{TM}}$, AquaplastTTM, Aquaplast Watercolors ${ }^{\mathrm{TM}}$ Moldable Thermo-pellets, Orfilight $^{\mathrm{TM}}$, Kay-splint, Polyflex ${ }^{\mathrm{TM}}$, San-Splint ${ }^{\mathrm{TM}}$ and many others), plaster of Paris, fiberglass, air splints, Neoprene, leather, Velcro strapping, Lycra, elastic band gloves and wooden dowels $[12,30]$.

Production and customization of the dynamic splint: When producing and customizing a dynamic splint the patient must be seated in a comfortable position with the elbow at 90 degrees and the hand in a functional position (wrist extension 
at 20-35 degrees, normal transverse arch, fingers flexed from 45-60 degrees and the thumb in abduction and opposition). A dynamic splint is cast on the hand in functional position i.e., between 30-35 degrees of wrist extension and when the muscle tone is higher; it is cast on the hand with wrist extension at 15 degrees to increase grip effort [31,32]. The length of the forearm component should be two-thirds of the forearm and the trough should cover a half of the transverse circumference of the forearm [22]. When the splint is cast on the hand it should be adjusted to avoid restricting the distal palmar crease to allow metacarpophalangeal (MCP) mobility [22].

In contrast, when customizing a static splint, the patient's hand should to be in a resting position (neutral wrist, fingers slightly flexed, the thumb extended and all hand components relaxed) $[22,33]$. The patient's anatomical center of the hand must be at the $3^{\text {rd }}$ metacarpal the point where all fingers converge 22 . The direction of pull of the fingers must be at a flexion of 10-30 degrees because all fingers flex obliquely with an exception of the $3^{\text {rd }}$ finger [22]. A study suggested that when a therapist is applying a splint, they must use personal protective equipment (PPE), non-sterile gloves and mask to avoid exposure to body fluids [33]. The therapist should monitor patient's vital signs, perform neurovascular assessment and remove any ornaments or clothing on the upper limb to be splinted [33].

All materials needed for splinting should be made available prior to the fitting process including the following e.g., scissors, springs, outrigger, tape measure, splint material, water bath oven with warm water, stockinette, cotton padding and elastic bandages or Velcro straps [33]. The dimensions of the area to be splinted may be drawn on the splinting material or paper before cutting [33]. A stockinette/padding material may be applied to the skin of the upper limb to prevent friction-related soft tissue injuries when applying the splint [33]. The splinting material should be warmed with hot water/gun until it is soft/ transparent and then dried by a towel to remove excess water. It is then cast on the patient's upper limb contours taking into consideration of the bony prominences while it is still wet or soft [28,33]. The splint edges should be smoothened to avoid friction-related injuries to the patient's hand [33]. When it dries/hardens; the splint is then secured to the hand by wrapping with elastic bandages or Velcro straps in a distal to proximal fashion [33]. The patient should be trained on the wearing schedule; cleaning schedule and to observe skin color changes on a daily basis [22]. The upper limb alignment; skin color; skin appearance; comfortability and pain level should be assessed after fitting the splint and adjusting the springs; bandages or straps [33].

The key features of the dynamic splint: A dynamic splint consists of a static base which forms the foundation of the splint and an outrigger; which is the mobile part consisting of levers; springs or pulleys [34,35]. It also consists a dynamic component to facilitate the splint mobility with the associated structures like finger springs, adjustable tensioner, finger lead mounts, digit caps, thumb spring and wrist mount areas [22,31]. A dynamic splint consists of 4 springs to fit 4 fingers and 1 spring to fit the thumb finger; which is connected to a modifiable tension builder to suit the patient's needs based on grasp and release actions [31]. The 4 finger lead mounts are placed on the distal part of the hand splint towards the finger tips [31]. The splint must also contain digit caps which prevents the movement of DIP finger joints and IP thumb joint [31]. By so doing; the digit caps allow efficient grasp and release actions of the hand [31]. Research demonstrates that patients who engage in more grasp and release activities show positive results in terms of decreased spasticity [31]. A typical design of a dynamic splint is shown in Figure 1 below.

Structurally, dynamic splints are designed to fit into the normal hand arches and contours; thereby providing comfort through inclusion of interior padding; maintaining axis of motion; enabling functional balance of unaffected muscles; and providing optimal stability with maximum mobility potential. They also free the palmar surface of the hand and offer large surface area for optimal pressure distribution in addition to exerting minimal stretch on muscles in the long-term period [22]. The splint also allows easy donning or doffing, lightweight, easy to clean and have a good cosmetic appearance [22]. A poorly designed dynamic splint may lead to complications such as joint deformity, soft tissue inflammation, pain, ischemia and pressure sores [36].

The mechanical forces within the dynamic splint: The mechanical efficiency and comfort of a dynamic splint can be increased by decreasing its tension levels [22]. Perpendicular traction and acceptable tension can be adjusted based on the feedback from the patient as it is adjusted to the hand [22]. The amount of tension on the springs depends on patient's condition [31]. If the aim is to improve grip strength or to learn to release objects, then more resistive springs are required; whereas to promote voluntary deactivation of hand flexors with increased hand extension activity; less resistive springs are used [31]. The tension and compression of the bandages/straps should be matched to the weight of the upper limb to produce the desired balance [22]. All horizontal forces in the dynamic splint should sum up to 0 because this avoids problems such as frictionrelated injuries; shearing and pressure induced soft tissuerelated injuries [22].

A study by da Silva et al. [36] investigated the amount of force/ tension required for each finger by designing a dynamic splint which can be connected to a dynamometer to allow forces measurement. They located the axis of rotation of the PIP joint of middle finger using Reuleaux method and then connected the dynamic splint to a dynamometer by Pesola ${ }^{\mathrm{TM}}(0-600$ grams of force scale and a $5 \mathrm{~g}$ resolution). Their research concluded that the spring tension increases at the beginning of PIP finger flexion and decreases when the flexion angle increases [36]. They further explained that the longer the finger; the greater the spring tension exerted on the tendon of the finger [36]. In a study by Willis et al. [37] it was suggested that a dynamic splint should offer a prolonged duration of inactive; joint precise stretching with small-load power.

\section{Current literature evidence}

The benefits of using dynamic splints with post-stroke patients: Based on prior studies; dynamic splints have been shown to enhance the capacity of post-stroke patient with plasticity of upper limbs to engage in task-specific training activities that involve grasp and release actions of the hand; which in turn promotes the plasticity of the brain because it 
imitates the functions of the hand [29]. Dynamic splints such as SaeboFlex allow effective active wrist and finger extensions to occur thereby improving the functionality of the hand [29]. In addition; they offer the biomechanical advantage of performing prehension grasp and release activities; reduces joint pains; protects the joints; and prevents edema when the hand is at rest [29]. Dynamic splints also enhance the stability of muscle tissues; restrict unnecessary motions which impair hand functions, compensate for the weak muscles and maintain the natural alignment of anatomical structures [22]. They have also been shown to decrease soft tissue adhesions; facilitate soft tissue remodeling and promote finger tendon gliding movements [22]. Radomski et al. also recognized that dynamic splints promote muscle balance; improve blood circulation and prevent muscle atrophy [22].

The effects of dynamic splinting on muscle tone and hand function: If the upper limb spasticity is left untreated; contractures develop due to abnormal shortening of the soft tissue structures spanning the joints such as the skin; ligaments; tendon; muscles and joint capsules [29]. An ideal dynamic splint should therefore facilitate hand mobility which stretches the muscles; tendons and ligaments to maintain their length thereby reducing spasticity [29]. Spasticity and impaired hand motor skills can be treated by harnessing the plasticity property of the brain through mass movement and task-oriented arm training [29]. The study by Pitts et al. [23] suggested that hand splints can be used to train motor learning and improve neural plasticity in the brain. When spasticity occurs together with immobilization; the resulting peripheral musculoskeletal tightness decreases the functional recovery of the hand [23]. They concluded that dynamic splints can improve the hand functional outcomes by maintaining the peripheral muscle and joint structures at a functional length [23].

When left in the immobilized state with the flexor synergy; the condition of the upper limbs progresses to a fibrotic state which triggers cross-sectional muscle bridge changes; loss of sarcomeres and development of early contractures [23]. In such conditions, splints provide a safe low-load force to the spastic muscles which facilitates muscle relaxation; maintains muscle length and prevent contractures [23]. In their study, Pitts et al. [23] suggested the Total End Range Time (TERT ) method should be used when fitting dynamic splints to stroke patients. This method involves the use of a biological creep (placing the spastic muscle tissue under constant safe stress) for 8 hours and a mechanical creep (placing a steady dynamic manipulation force from a device) for 2 hours [23]. Research confirms that muscle activation when the muscle is at a low-load stretch can reduce hypersensitivity of the stretch reflex [31].

Literature evidence for the effectiveness of dynamic splints: In a previous study [32], the use of dynamic splints on the upper limb function was investigated in 8 post-stroke patients. The patients wore the splints for 45 minutes a day; 5 times per week and for a 6 -week period. The results of the study showed that at the end of the treatment period, patients had improved hand function; high motivation and self-regulation [32]. Another study Kimbler et al. [38] investigated the effectiveness of dynamic splints on the contractures of the hands of patients with spinal cord injuries. The patients wore the splints for 45 minutes in each hand twice a day; and were given occupational therapy treatments 5 times a week for a period of 3 months. Their findings revealed that a dynamic splint can reduce spasticity and improve the functionality of the hand [38]. Several other studies have also reported that dynamic splinting reduces spasticity and prevents contractures of the upper limb muscles [20,39,40].

A study by Prakash et al. [41] investigated the effect of dynamic splints on the deltoid muscle activity in stroke patients using functional tasks. Thirty participants were divided into 2 groups; control group (performed functional tasks without the splint) and the treatment group (performed functional tasks using a splint) for 30 minutes per day, 3-4 times per week, for a period of 4 weeks. They found that there was significant improvement in hand functionality in the group that used the dynamic splint compared to the control group [41]. Furthermore, Chang et al. [40] the effects of dynamic splints on the muscle strength and hand function in post-stroke patients were demonstrated by another study [40]. In this study a comparison of hand functionality was made between patients received conventional therapy and those that received dynamic splints for 3 months. The results indicated that wearing a dynamic splint that is customized for a home-use rehabilitation program in addition to receiving a hospital treatment program may improve hand functions reduce spasticity and enhance muscle strength of hemiplegic hands [40].

Literature evidence for the ineffectiveness of dynamic splints: A study by Eghlidi et al. compared the effectiveness of dynamic and static splints in reducing spasticity of the hand in post-stroke patients [42]. Thirty-one stroke patients were randomly divided into 3 treatment groups, static splint, dynamic splint and a control group. Participants in the intervention groups wore customized splints for 6 hours per day, 5 days a week and for a period of 3 months [42]. The Modified Ashworth Scale (MAS) and Electromyography (EMG) tests were used to measure wrist spasticity at the baseline and after 3 months. Their results showed that the splints did not induced any significant effect on hand spasticity [42]. In a randomized controlled trial by Lannin et al. [43], the effect of a static neutral splint on hand spasticity was investigated. Sixty-three post-stroke patients were divided into control and treatment groups. The neutral static splints were fitted while the hands of the participants were in the neutral and slight extension position for 9-12 hours per day, overnight, for a period of 4 weeks. Similar to the study by Eghlidi et al. they found that the static splints did not reduce hand spasticity in stroke patients [43].

Collectively, studies by Rose et al., Langlois et al. and Basaran et al. which were performed to evaluate the effectiveness of splints on reducing spasticity in upper limbs using hand resting splints for 4-6 weeks, showed that the splints did not reduce spasticity [39]. Moreover, several studies have suggested that static splinting might not reduce spasticity in most cases and but can only be used to prevent contractures [44-46]. Interestingly the use of static splints may cause many complications and should be avoided according to a previous randomized controlled trial [43].

Critical issues arising from previous studies that utilized 


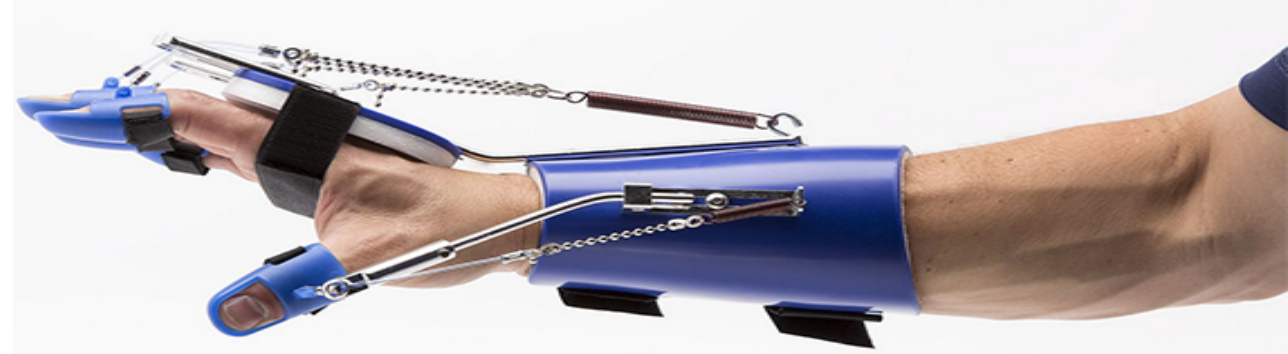

Figure 1. This figure shows a type of a dynamic splint called Saebo Flex [29].

the dynamic splint: A study by Gambhir reported that most therapists in the current era prefer dynamic splints to static splints as they produce superior results in patients with hand spasticity [12]. With regard to the controversies surrounding the effectiveness of dynamic splints, Franck et al. suggested that the hand recovery observed in post-stroke patients when dynamic splints were applied was suspected to be a result of spontaneous recoveryspontaneous recovery since his patients were in the sub-acute stage of stroke [32]. In contrast, Naro et al. postulated that non-pharmacological treatments; including dynamic splints, reduce spasticity by inducing changes to the viscoelastic properties of connective tissues [20]. This mechanism may explain the positive results of dynamic splints reported in some studies; although additional molecular and histological studies are required to validate this concept. Moreover, it has been suggested that future studies should consider the etiology of spasticity, age of patient and severity of the spasticity as they can predict the recovery process and choice of treatment strategies [20].

Furthermore, Kimbler et al. suggested that progressive tension changes the end range, and therefore dynamic splint tension should be adjusted to challenge the new end range [38]. This implies that dynamic splints can be designed to suit the progressive increases in the range of motion of the wrist. Mechanistically, Foley et al. suggested that training and treatment in the first 4 weeks after a stroke event could result in upregulation of growth promoting factors thereby reducing spasticity and increasing hand function [47]. Indeed, the usage of dynamic splints at hospital settings with a home program produces positive results as reported by Chang and Lai [40]. However, a major drawback of their study is that they did not include a control group. Thus; this limits the interpretation of their findings. A study by Prakash stated that the patients may have experienced improved outcomes due to the motor cortex priming and reorganization [41]. But in their study they did not consider the stroke duration of their participants; which is a key determinant of recovery. It is worthy to note that the duration of treatment may influence the recovery outcomes. For instance, Lannin et al. concluded that the absence of positive outcomes after dynamic splints application in their investigation was due to the short treatment duration of 4 weeks [43]. Moreover, the participants were not blinded which affects the validity of their outcomes since the lack of blinding may influence the patients' responses to subjective outcome measures.

\section{Conclusions and key issues emerging from this review}

Based on the datasets reviewed above; it can be noted that dynamic splints might not have been effective in reducing hand spasticity because spasticity does not occur alone; but it is usually accompanied with other conditions such as pain; fibrosis, contracture, movement disorders and muscle weakness. For the studies where, dynamic splints produced positive effects, it could have been the viscoelastic changes in the muscles or spontaneous recovery of the patient. Further studies are advocated to develop standardized and conventional methods for the application of dynamic splints. Importantly, assessment tools or devices which can accurately measure the intensity of spasticity of the hand before prescribing a splint should be explored. Further research should also investigate whether dynamic splints reduce spasticity directly or indirectly through other physiological processes and should take serious consideration of the etiology of spasticity, age of patient, stage of stroke and spasticity severity as they can interfere with results. There is also need to develop standardized measurement device embedded within the dynamic splint to show the amount of force/tension directed to the fingers and to measure the direct effect of the splint on the muscular tissues. Clinical trials should be performed to investigate whether dynamic splints are effective in the acute or chronic stages of post-stroke patients, combining structured home-program and the precise duration of use. Future studies might also consider investigating the effect of a progressive dynamic splint (which can be adjusted the angle of the static base after certain intervals) on hand spasticity.

\section{Acknowledgements}

This study was funded by the Jiangsu Youth Medical Talent Fund (Jiangsu Provincial Medical Youth Talent QNRC2016376) and Xuzhou City Medical Young Reserve Talents Project Grant (Xuzhou Medical Young Talents Project 2016015).

\section{Declaration of Conflicting Interests}

All authors declare that no conflict of interest exists regarding that writing and publication of this manuscript.

\section{References}

1. Bartels MN, Duffy CA, Beland HE. Pathophysiology, medical management and acute rehabilitation of stroke survivors ( $4^{\text {th }}$ ed.). Elsevier Ltd. 2016.

2. Thibaut A, Chatelle C, Ziegler E, et al. Spasticity after stroke: Physiology, assessment and treatment. Brain Inj. 2013;27(10):1093-105.

3. Stroke Association. State of the nation stroke statistics. Stroke Assoc Resour Sheet. 2017;pp:1-37.

4. Johansson G. Clinical and kinematic assessments of upper limb function in persons with post-stroke symptoms. 2015;pp:1-90. 
Citation: Bianca C, Machuki JO, Chen W, et al. A dynamic splint for the treatment of spasticity of the hand after stroke-recognition of its design, functionality and limitations: A narrative review article. J Neurol Neurorehabil Res. 2018;3(2):1-7.

5. Leo A, Naro A, Molonia F, et al. Spasticity management: The current state of transcranial neuromodulation. PMR. 2017;9(10):1020-29.

6. Atchison BJ, Dirette DK. Conditions in occupational therapy: Effect on occupational performance $4^{\text {th }}$ edition. Lippincott Williams \& Wilkins. 2012;2:1.

7. Raghavan P. Upper limb motor impairment post stroke. Phys Med Rehabil Clin N Am. 2015;26(4):599-610.

8. Lance J. What is spasticity? Medline. 1990;10(1):606.

9. Ivanhoe C, Reistetter T. Spasticity: The misunderstood part of the upper motor neuron syndrome. Am J Phys Med Rehabil. 2004;pp:3-9.

10. Rowland T, Cooke D, Gustafsson L. Role of occupational therapy after stroke. Ann Indian Acad Neurol. 2008;11:S99107.

11. Dyna Splint. Dynamic splints: The industry leading dynamic splint. 2018.

12. Gambhir PK. Evidence base of stroke and hand splinting. Int J Sci Res. 2016;5(2):2014-2017.

13. Sommerfeld DK, Eek EU, Svensson A, et al. Spasticity after stroke: Its occurrence and association with motor impairments and activity limitations. Stroke. 2003;pp:134-41.

14. Pascual-Leone A. Arm function after stroke: From physiology to recovery. Semin Neurol. 2005;25(4):384-395.

15. Bhakta BB. Management of spasticity in adults. Aust J Physiother. 2009;55(4):290.

16. Xu J, Ejaz N, Benjamin H, et al. Recovery of hand function after stroke: Separable systems for finger strength and control. CSH Neuroscience. 2016.

17. Kheder A, Nair KPS. Spasticity: Pathophysiology, evaluation and management. Pract Neurol. 2012;12(5):289-298.

18. Rodrigues A. Immediate effect of acupuncture on poststroke patients with spastic upper limb hemiparesis ana rita rebelo tavares rodrigues 2014 immediate effect of acupuncture on poststroke patients with spastic upper limb hemiparesis. 2014.

19. Trompetto C, Marinelli L, Mori L, et al. Pathophysiology of spasticity: Implications for neurorehabilitation. Biomed Res Int. 2014.

20. Naro A, Leo A, Russo M, et al. Breakthroughs in the spasticity management: Are non-pharmacological treatments the future?. J Clin Neurosci. 2017;39:16-27.

21. Wissel J, Schelosky LD, Scott J, et al. Early development of spasticity following stroke: A prospective, observational trial. J Neurol. 2010;257(7):1067-72.

22. Radomski M, Latham CA. Occupational therapy for physical dysfunction $6^{\text {th }}$ edition. 2008;16:17.

23. Pitts DG, O'Brien SPO. Splinting the hand to enhance motor control and brain plasticity. Top Stroke Rehabil. 2008;15(5):456-67.
24. Taylor CL, Schwarz RJ. The anatomy and mechanics of the human hand. 1955;pp:22-35.

25. Kumar A, Mundra TS, Kumar A. Hand Anatomy. 2018.

26. Orthopod. A patient's guide to hand anatomy. 2018.

27. Ghadiali MT. Normal hand anatomy. Multimedia Health Education. 2018.

28. Asplund C. Splints and casts: Indications and methods. American Family Physician. 2014;80(5):491-99.

29. Saebo. Benefits of rehabilitation gloves and dynamic splints for stroke recovery. Rehab Solutions from Saebo.2016.

30. Spro-Fit. Hand Positioning Splints. 2018;pp:54-55.

31. Hoffman HB, Blakey GL. New design of dynamic orthoses for neurological conditions. Neuro Rehabilitation. 2011;28:55-61.

32. Franck JA, Timmermans AAA, Seelen HAM. Effects of a dynamic hand orthosis for functional use of the impaired upper limb in sub-acute stroke patients: A multiple single case experimental design study. Technology and Disability. 2013;25:177-87.

33. Schub E, Balderrama D, DeVesty G, et al. Nursing practice and skill, splints: Applying to an extremity. Cinahl Inf Syst. 2017.

34. AHM. Dynamic splinting devices. 2016;pp:1-11.

35. Spro0Fit. Hand splinting catalogue. 2018;p:32.

36. Da Silva SNP, Mattar RJ, Bolliger RN, et al. Measurement of the flexing force of the fingers by a dynamic splint with a dynamometer. Clinics. 2005;60(5):381-8.

37. Willis FB, Fowler B. Longitudinal outcomes following a randomized controlled trial of dynamic splint stretching for carpal tunnel syndrome. Hand. 2016;11(3).

38. Kimbler TS, Willis FB. Dynamic splinting for pronation contracture following a spinal cord injury. Hand Therapy. 2010;15(1):20-2.

39. Foley N, Teasell R, Cpsych JJ, et al. Upper extremity interventions. Evidence-Based Rev Stroke Rehabil. 2011.

40. Chang WD, Lai PT. New design of home-based dynamic hand splint for hemiplegic hands: A preliminary study. J Phys Ther Sci. 2015;27(3):829-31.

41. Prakash J, Mondam S, Srinivasan M, et al. Effectiveness of dynamic wrist splint on deltoid activity using functional task practice in post stroke patients. Int $\mathrm{J}$ Physiother Res. 2013;(2):30-3.

42. Eghlidi J, Elyaspour D, Tabatabaee S. et al. Comparison of the effect of dynamic and static splints on wrist spasticity in post-stroke patients. JRUMS. 2011;10(1):35-45.

43. Lannin NA, Cusick A, McCluskey A, et al. Effects of splinting on wrist contracture after stroke: A randomized controlled trial. Stroke. 2007;38(1):111-6.

44. Blackmore AM, Garbellini SA, Buttigieg P, et al. A systematic review of the effects of soft splinting on upper 
limb function in people with cerebral palsy an AACPDM Evidence Report Initial Publication In. Aacpdm Org. 2006.

45. Teasell R, Foley N, Mehta S, et al. Upper extremity interventions. Evidence-Based Rev Stroke Rehabil. 2013;pp:1-163.
46. Choi JB, Ma SR, Song BK. The effect of resting hand splint on hand pain and edema among patients with stroke. J Ecophysiol Occup Heal. 2016;16(1-2):37-41.

47. Langhorne P, Bernhardt J, Kwakkel G. Stroke rehabilitation. Lancet. 2011;377(9778):1693-702.

\section{*Correspondence to:}

Chen Wei

Xuzhou Rehabilitation hospital, 10 Kuizhong road,

Xuzhou, Jiangsu, P.R.China

Tel : +8618952172339

E-mail: chenwei2339@163.com 